\title{
Improvement of Water and Wastewater Treatment Process Using Various Sound Waves-A Consideration from the Viewpoint of Frequency
}

\author{
Akira Hiratsuka $^{1 *}$, Yugo Tomonaga ${ }^{2}$, Yoshiro Yasuda ${ }^{3}$, Ryoji Tsujino ${ }^{4}$ \\ ${ }^{1}$ Department of Civil Engineering, Osaka Sangyo University, Osaka, Japan \\ ${ }^{2}$ National Museum of Ethnology, Osaka, Japan \\ ${ }^{3}$ University of Hyogo, Kobe, Japan \\ ${ }^{4}$ SetsunanUniversity, Osaka, Japan \\ Email: ${ }^{*}$ hiratuka@ce.osaka-sandai.ac.jp
}

Received 13 September 2014; revised 12 October 2014; accepted 5 November 2014

Copyright (C) 2014 by authors and Scientific Research Publishing Inc.

This work is licensed under the Creative Commons Attribution International License (CC BY). http://creativecommons.org/licenses/by/4.0/

C. (i) Open Access

\begin{abstract}
Application of sound waves is one of the novel techniques for the improvement of water treatment process. In this study, various sound waves such as 1) ultrasonic wave, 2) music box, and 3) windbell were irradiated to water and wastewater for removing contaminants such as nitrate, phosphorus and BOD/COD. As a result, a possibility of improvement of water and wastewater treatment process using sound waves with various frequencies was proposed.
\end{abstract}

\section{Keywords}

Sound Waves (Frequency), Total Hardness $\left(\mathrm{Ca}^{2+}, \mathrm{Mg}^{2+}\right)$, Nitrogen (N), Phosphorus (P), Water Treatment

\section{Introduction}

Several anthropogenic processes resulting from haphazard urbanization have mounted heavy stress on water quality of urban basins in underdeveloped and developing countries (Pathak et al., 2011) [1]. And then, river water is more susceptible to pollutants and is highly polluted as a result of rapid urbanization, that is, increased loadings of diffused pollutants and exploitation of sources of rivers by foreign enterprises (multinational corporations etc.). Therefore, it is very important to protect and conserve source of a river. Regarding pollution 
sources, unplanned urban growth, poor sewerage and waste disposal system and fertilizers and other chemicals are considered. The unplanned and haphazard urbanization in the valley has led to poor drainage and sanitation. The solid wastes and disposals along the riverbanks and the untreated sewage and wastewaters from domestic and industrial areas are directly discharged into the rivers rendering them as open seers [1]. Health is related to nitrate content in drinking water. It is said that Blue-baby syndrome in infants etc. occurs due to nitrate in drinking water. The role that nitrates play in diabetes etiology may be related to the endogenous formation of diabetogenic nitrosamines. Previous studies [2] have shown that toxic doses of nitrosamines or N-nitroso compounds can cause diabetes via the generation of free radicals that damage the insulin-producing $\beta$-cells of the pancreas. Although source protection is more important and cost-effective than remediation and treatment, the water sources already contaminated have to be treated to make drinking water safe. Therefore, an efficient and costeffective treatment technique is required for the removal of nitrate from Drinking water. By the way, there are three main types of water treatment systems at present. The first one is a "rapid sand filtration (physicochemical purification system)", the second one is a "slow sand filtration (ecological purification system)" and the third one is a "natural filtration (natural purification system)". However, the removal of the nitrate is impossible under the present water purification system even in Japan. Although it is possible for only reverse osmosis membrane (ROM) to remove the nitrate- $\mathrm{N}$, this ROM is very expensive. Therefore, it is very difficult to introduce this ROM to the present system additionally from a cost-effectiveness perspective in the future. As mentioned above, the most widely applied existing water treatment processes have some purification limitation and application problems such as high cost, ineffectiveness for the removal of some pollutants, operation problems and the generation of toxic secondary pollutants (Gaya and Abdulla, 2008) [3]. Such a limitation can be eliminated by the application of innovative techniques including sound waves (Chong et al., 2010) [4]. The application of sound waves is one of the novel techniques for the improvement of water treatment process. In this study, various sound waves such as 1) ultrasonic waves, 2) music box, and 3) wind-bell were irradiated to water and wastewater for removing contaminants such as nitrate, phosphorus and BOD/COD.

\section{Experimental Methods}

In previous study [5], an examination for the improvement of water treatment process using ultrasonic waves $(28 \mathrm{KHz})$ was carried out. As a result, It was confirmed that ultrasonic wave affects water quality such as content of calcium (Ca), magnesium (Mg), namely, hardness, sodium (Na) and potassium (K). In addition to those, the possibility of the complete removal of impurities (BOD, COD) was supposed by the irradiation. The experimental results confirmed that the ultrasonic waves improved the water treatment process significantly by accelerating the reaction. Therefore, this time we examined a possibility of an effect of improvement of water treatment process using various sound waves with different frequency in addition to the ultrasonic waves mentioned above. Three kinds of sound waves such as 1) ultrasonic wave (35 KHz), 2) Switzerland music box (3.75 Hz $102 \mathrm{KHz})$, and 3) Japanese wind-bell $(1500 \mathrm{~Hz}-8.5 \mathrm{KHz})$ were irradiated to drinking water, forest water and sea water for water softening process as for total hardness and other contaminants removal such as nitrate, phosphorus and BOD/COD etc. Figure 1 and Figure 2 show a frequency range of sound waves and appearance of the sound boxes, respectively.

Details of three types of the sound system are as follows.

1) Ultrasonic system

A specification of the ultrasonic cleaner is as follows: Model: SK5210LHC, Frequency:35 KHz/53KHz, Power : $200 \mathrm{~W}$, Output adjust: 40\% - 100\%, Heater: $20^{\circ} \mathrm{C}-60^{\circ} \mathrm{C} / 270 \mathrm{~W}$, Dimensions W $\left.\times \mathrm{D} \times \mathrm{H}\right):$ Inter: $330 \times$ $300 \times 150$ mm, Exter: $360 \times 330 \times 310$ mm, Capacity: 10 L, Timer: 1 - 199 min., Drain cock: Yes, Accessories: Drain board, Power supply: AC 220 V 50/60 Hz or AC 100 - 110 V 50/60 Hz with transformer, Maker: Shinka Industry Co., Ltd., Japan.

2) Music box system

A specification of the music box is as follows: Model: Cylinder Music Box "Rose”, Frequency: 3.75 Hz - 102 KHz, 72-note comb, Song title: Canon (Composer: Johann Pachelbel), Playing time: 15.41 min. (Playing was continuously conducted twice in the experiment.), Maker: REUGE Manufacture Co., Ltd., Switzerland.

3) Wind-bell system (called “Furin”)

A specification of the Japanese wind-bell is as follows: Model: Nambu-Furin, Material: Made of metal, Frequency: $1.5 \mathrm{KHz}-8.5 \mathrm{KHz}$, This wind-bell hung inside a basket to be played by the wind (an electric fun). (In 


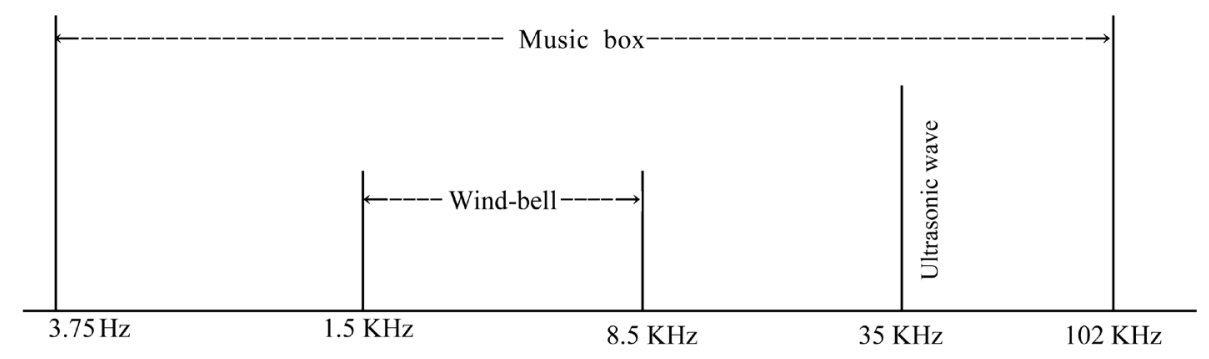

Figure 1. Frequency range of music box, wind-bell and ultrasonic wave.

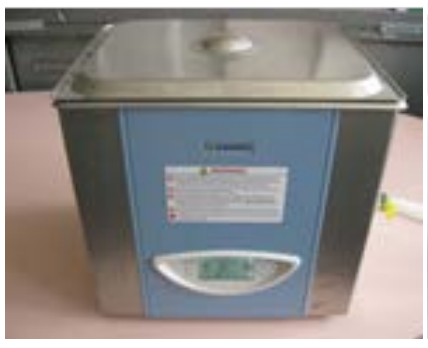

(a)

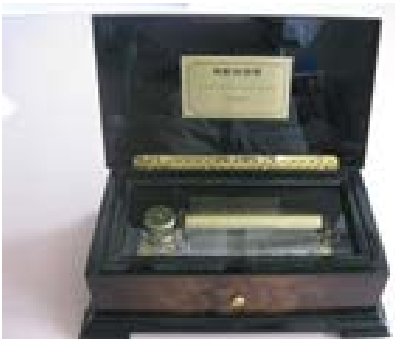

(b)

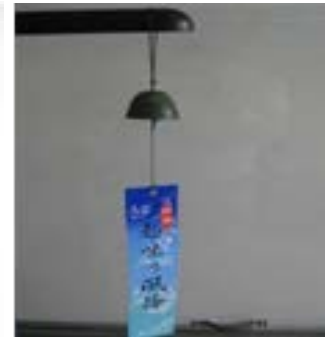

(c)

Figure 2. Three types of sound boxes used in the experiment. (a) Ultrasonic system (35 KHz);

(b) Music box system (3.75 Hz - $102 \mathrm{KHz}$ ); (c) Wind-bell system $(1500 \mathrm{~Hz}-8.5 \mathrm{KHz})$.

general, Japanese wind-bell is often made of metal or glass, and some of the famous Japanese Furins are "EdoFurin" from Tokyo and "Nambu-Furin” from Iwate prefecture.)

\subsection{Experimental Condition}

In this study, the standpoint is to understand how the Hardness $\left(\mathrm{Ca}^{2+}, \mathrm{Mg}^{2+}\right)$, nitrate- $\mathrm{N}$, phosphorus (P), and $\mathrm{BOD} / \mathrm{COD}$, those are causal substances of eutrophication and organic contamination are decreased in each water sample by irradiating ultrasonic wave, music box and wind-bell. The experimental conditions of these three types of sound boxes used in this experiment are as follows: 1) Ultrasonic wave: Frequency: $35 \mathrm{KHz}$, Irradiation time: 30 min., 2) Music box: Frequency: $3.75 \mathrm{~Hz}$ - $102 \mathrm{KHz}$, Irradiation time: $30 \mathrm{~min}$., 3) Wind-bell: Frequency: $1500 \mathrm{~Hz}-8.5 \mathrm{KHz}$, Irradiation time $30 \mathrm{~min}$. Drinking water (Hard water/Soft water), Forest water (Upper reach/ Middle reach/Lower reach) and Sea water were used as test samples (see Table 1). The analysis of water qualities was focusing nitrate, phosphorus and BOD/COD.

\subsection{Analytical Method of Water Quality}

The analysis of the water quality was conducted using simplified water quality analysis kit made by Kyoritsu chemical-check lab., corp., Japan. The kit was used for examining Total Hardness, $\mathrm{pH}, \mathrm{COD}, \mathrm{Mg}^{2+}, \mathrm{Ca}^{2+}, \mathrm{SiO}_{2}$, $\mathrm{NH}_{4}^{+}, \mathrm{NO}_{2}^{-}, \mathrm{NO}_{3}^{-}, \mathrm{PO}_{4}^{3-}, \mathrm{BOD}$. Furthermore, ICP emission spectrometry was used to analyze for $\mathrm{Na}^{+}$and $\mathrm{K}^{+}$, respectively.

\section{Experimental Results}

\subsection{Improvement of Water Quality Using Ultrasonic Wave}

An improvement of water quality using ultrasonic wave with frequency $35 \mathrm{KHz}$ is shown in Table 2.

Removal rate of each substance by the ultrasonic wave is shown in Figures 3-8. The ultrasonic wave is very effective for improving the water quality for especially drinking water (hard water/soft water) and forest water. The results are as follows.

1) Nitrate: As for the removal rate of $\mathrm{NO}_{2}^{-}$and $\mathrm{NO}_{3}^{-}$concerning to the three samples such as the sea water, drinking water (soft water) and the forest water, high values ( $>50 \%)$ were obtained, respectively.

2) Phosphorus: $\mathrm{PO}_{4}^{3-}$ was also confirmed to decrease larger than $50 \%$ in the forest water (2 and 3$)$. 
Table 1. Description of test water samples.

\begin{tabular}{crcc}
\hline Test Sample & & Content & Country Made \\
\hline Drinking Water 1 & & Hard Water & Nancy City, France \\
Drinking Water 2 & (A) & Soft Water & Kameoka City, Japan \\
Forest Water 1 & (B) & Soft Water & Melbourne City, Australia \\
Forest Water 2 & & Upper Reach & Kameoka City, Japan \\
Forest Water 3 & & Middle Reach & Kameoka City, Japan \\
Sea Water & & Lower Reach & Kameoka City, Japan \\
\hline
\end{tabular}

Table 2. Change of chemical substances in the water after ultrasonic wave irradiation.

\begin{tabular}{|c|c|c|c|c|c|c|}
\hline & Sea Water & $\begin{array}{l}\text { Drinking Water } 1 \\
\text { (Hard Water) }\end{array}$ & $\begin{array}{l}\text { Drinking Water 2(A) } \\
\text { (Soft Water) }\end{array}$ & $\begin{array}{l}\text { Forest Water } 1 \\
\text { (Upper Reach) }\end{array}$ & $\begin{array}{c}\text { Forest Water } 2 \\
\text { (Middle Reach) }\end{array}$ & $\begin{array}{l}\text { Forest Water3 } \\
\text { (Lower Reach) }\end{array}$ \\
\hline $\begin{array}{c}\text { Sampling } \\
\text { Point } \\
\text { Sub-Stances }\end{array}$ & $\begin{array}{l}\text { Kobe City, } \\
\text { Japan }\end{array}$ & $\begin{array}{l}\text { Nancy City, } \\
\text { France }\end{array}$ & $\begin{array}{l}\text { Kameoka City, } \\
\text { Japan }\end{array}$ & $\begin{array}{l}\text { Kameoka City, } \\
\text { Japan }\end{array}$ & $\begin{array}{l}\text { Kameoka City, } \\
\text { Japan }\end{array}$ & $\begin{array}{c}\text { Kameoka City, } \\
\text { Japan }\end{array}$ \\
\hline $\mathrm{TH}(\mathrm{mg} / \mathrm{L})$ & $50 \rightarrow 40$ & $1468 \rightarrow 100$ & $30 \rightarrow 10$ & $10 \rightarrow 0$ & $20 \rightarrow 0$ & $50 \rightarrow 20$ \\
\hline $\mathrm{pH}$ & $9.0 \rightarrow 9.0$ & $7.4 \rightarrow 7.4$ & $7.5 \rightarrow 7.0$ & $7.5 \rightarrow 7.5$ & $7.5 \rightarrow 7.0$ & $8.0 \rightarrow 7.5$ \\
\hline $\mathrm{COD}(\mathrm{mg} / \mathrm{L})$ & $8 \rightarrow 4$ & $5 \rightarrow 0$ & $5 \rightarrow 0$ & $0 \rightarrow 0$ & $0 \rightarrow 0$ & $4 \rightarrow 4$ \\
\hline $\mathrm{Ca}^{2+}(\mathrm{mg} / \mathrm{L})$ & $35 \rightarrow 25$ & $468 \rightarrow 40$ & $35 \rightarrow 25$ & $12.5 \rightarrow 12.5$ & $25 \rightarrow 12.5$ & $50 \rightarrow 25$ \\
\hline $\mathrm{Mg}^{2+}(\mathrm{mg} / \mathrm{L})$ & $31 \rightarrow 8.2$ & $74.5 \rightarrow 30$ & $8.2 \rightarrow 4.1$ & $0 \rightarrow 0$ & $8.2 \rightarrow 4.1$ & $8.2 \rightarrow 4.1$ \\
\hline $\mathrm{SiO}_{2}(\mathrm{mg} / \mathrm{L})$ & $0.5 \rightarrow 0.5$ & $8 \rightarrow 5$ & $100 \rightarrow 10$ & $15 \rightarrow 5$ & $15 \rightarrow 15$ & $15 \rightarrow 15$ \\
\hline $\mathrm{NH}_{4}^{+} \quad(\mathrm{mg} / \mathrm{L})$ & $0.65 \rightarrow 0.26$ & $0.26 \rightarrow 0.26$ & $0.26 \rightarrow 0.26$ & $0.26 \rightarrow 0$ & $0.26 \rightarrow 0.26$ & $0.26 \rightarrow 0.26$ \\
\hline $\mathrm{NO}_{2}^{-} \quad(\mathrm{mg} / \mathrm{L})$ & $0.33 \rightarrow 0$ & $0 \rightarrow 0$ & $0.033 \rightarrow 0$ & $0 \rightarrow 0$ & $0.033 \rightarrow 0.033$ & $0.066 \rightarrow 0.033$ \\
\hline $\mathrm{NO}_{3}^{-} \quad(\mathrm{mg} / \mathrm{L})$ & $8.6 \rightarrow 0$ & $2.2 \rightarrow 2.2$ & $8.6 \rightarrow 4.3$ & $0 \rightarrow 0$ & $0.86 \rightarrow 0.86$ & $4.3 \rightarrow 2.2$ \\
\hline $\mathrm{PO}_{4}^{3-} \quad(\mathrm{mg} / \mathrm{L})$ & $0 \rightarrow 0$ & $0 \rightarrow 0$ & $0 \rightarrow 0$ & $0 \rightarrow 0$ & $0.15 \rightarrow 0.06$ & $0.3 \rightarrow 0.15$ \\
\hline BOD (mg/L) & $3 \rightarrow 0$ & $2 \rightarrow 0$ & $3.5 \rightarrow 0$ & $1 \rightarrow 0$ & $1.5 \rightarrow 0$ & $2.5 \rightarrow 0$ \\
\hline $\mathrm{Na}^{+}(\mathrm{mg} / \mathrm{L})$ & $7900 \rightarrow 8800$ & $25 \rightarrow 27$ & $5.9 \rightarrow 7.5$ & $4.4 \rightarrow 4.7$ & $4.3 \rightarrow 5.6$ & $6.0 \rightarrow 6.6$ \\
\hline $\mathrm{K}^{+}(\mathrm{mg} / \mathrm{L})$ & $410 \rightarrow 430$ & $5.2 \rightarrow 5.8$ & $1.4 \rightarrow 1.6$ & $0.7 \rightarrow 0.7$ & $0.7 \rightarrow 0.8$ & $2.0 \rightarrow 2.0$ \\
\hline
\end{tabular}

3) BOD/COD: BOD and COD drastically decreased especially in drinking water (hard water/softwater) and forest water.

4) $\mathrm{Ca}^{2+}, \mathrm{Mg}^{2+}$ and $\mathrm{TH}$ : The removal rate of $\mathrm{Ca}^{2+}$ and $\mathrm{Mg}^{2+}$ was also large (>50\%) in the drinking water (hard water/soft water). Along with these, TH also drastically decreased. Here, TH means total hardness defined as content of $\mathrm{Ca} \times 2.5$ plus $\mathrm{Mg} \times 4.1$.

5) $\mathrm{Na}^{+}, \mathrm{K}^{+}$and $\mathrm{NH}_{4}^{+}$: They showed very few change.

\subsection{Improvement of Water Quality Using Switzerland Music Box}

An improvement of water quality using sounds by Switzerland music box with frequency $3.75 \mathrm{~Hz}$ to $102 \mathrm{KHz}$ is shown in Table 3.

Removal rate of anions by the ultrasonic wave is shown in Figures 9-11. From Table 3 and Figures 9-11, Nitrate $\left(\mathrm{NO}_{2}^{-}, \mathrm{NO}_{3}^{-}\right)$, Phosphorus $\left(\mathrm{PO}_{4}^{3-}\right), \mathrm{BOD} / \mathrm{COD}$ and others $\left(\mathrm{Ca}^{2+}, \mathrm{Mg}^{2+}, \mathrm{Na}^{+}, \mathrm{K}^{+}, \mathrm{NH}_{4}^{+}\right)$show almost the same removal rates as ultrasonic wave. 


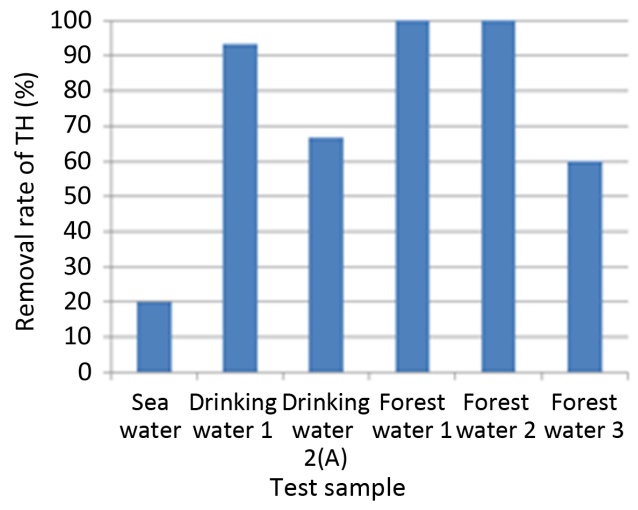

Figure 3. Removal rate of TH.

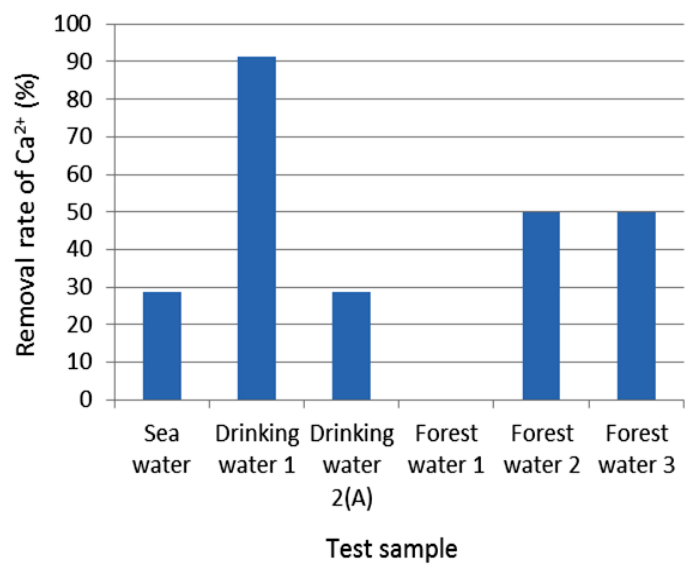

Figure 4. Removal rate of $\mathrm{Ca}^{2+}$.

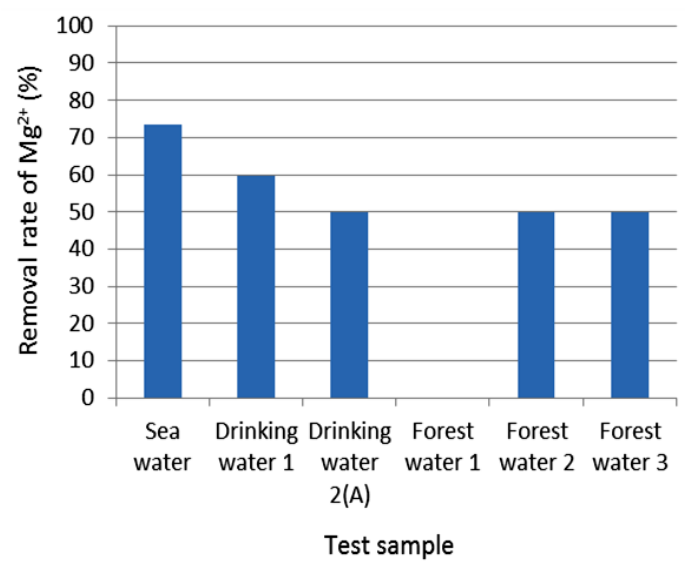

Figure 5. Removal rate of $\mathrm{Mg}^{2+}$.

\subsection{Improvement of Water Quality Using Japanese Wind-Bell}

Third, an improvement of water quality using sounds by Japanese wind-bell with frequency $1500 \mathrm{~Hz}$ to $8.5 \mathrm{KHz}$ in Table 4. Removal rate of anions by the ultrasonic wave are shown in Figures 12-14. From Table 4 and Figures 12-14, this case also shows almost the same tendency of the removal rate of the quality of water concerning to Nitrate $\left(\mathrm{NO}_{2}^{-}, \mathrm{NO}_{3}^{-}\right)$, Phosphorus $\left(\mathrm{PO}_{4}^{3-}\right), \mathrm{BOD} / \mathrm{COD}$ and others $\left(\mathrm{TH}, \mathrm{Ca}^{2+}, \mathrm{Mg}^{2+}, \mathrm{Na}^{+}, \mathrm{K}^{+}, \mathrm{NH}_{4}^{+}\right)$as that of the ultrasonic wave. 


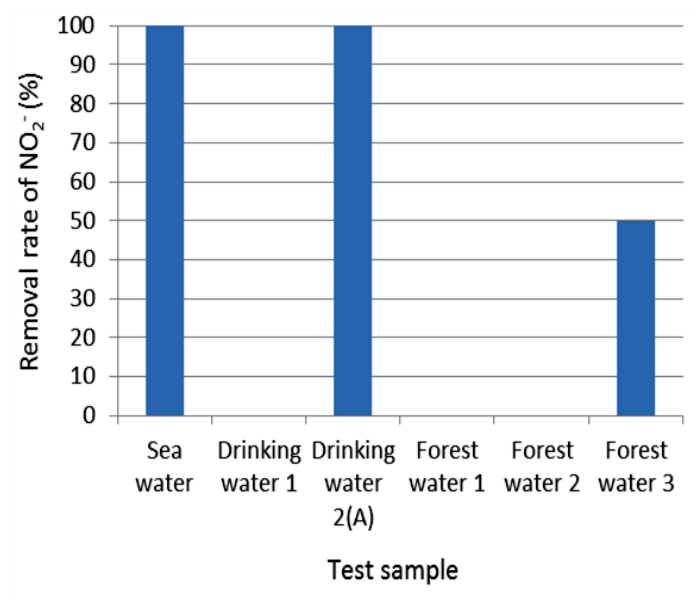

Figure 6. Removal rate of $\mathrm{NO}_{2}^{-}$.

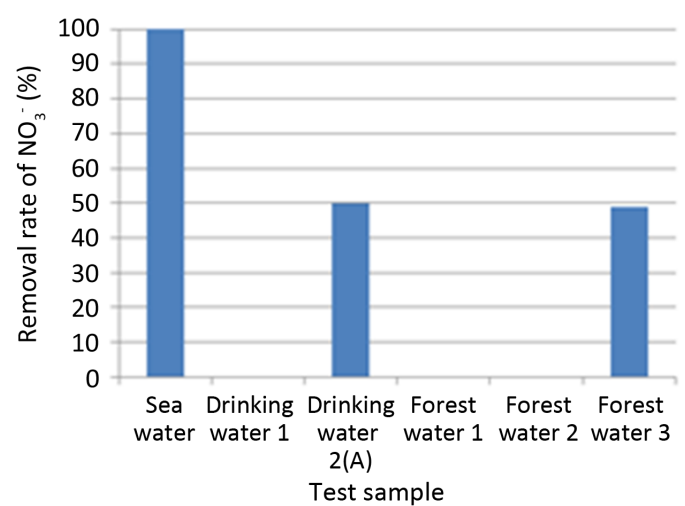

Figure 7. Removal rate of $\mathrm{NO}_{3}^{-}$.

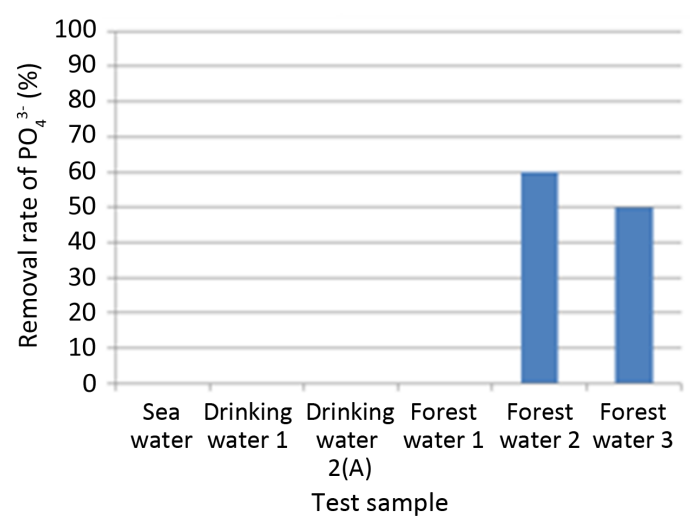

Figure 8. Removal rate of $\mathrm{PO}_{4}^{3-}$.

\section{Discussion}

\subsection{Chemical Reaction under Sound Waves}

All measured results are shown in Tables 2-4. The $\mathrm{pH}$ values show little change after irradiating in all cases. Furthermore, the decrease of $\mathrm{Ca}^{2+}, \mathrm{Mg}^{2+}$ and $\mathrm{NO}_{2}^{-},\left(\mathrm{NO}_{3}^{-}\right), \mathrm{PO}_{4}^{3-}$ were observed. This estimates that these ions react to each other to make neutral chemical compounds. That is, following reactions were occurred. 
Table 3. Change of chemical substances in the water by Switzerland music box.

\begin{tabular}{|c|c|c|c|c|c|c|c|}
\hline & Sea Water & $\begin{array}{l}\text { Drinking Water } \\
1 \text { (Hard Water) }\end{array}$ & $\begin{array}{l}\text { Drinking Water } \\
\text { 2(A) (Soft Water) }\end{array}$ & $\begin{array}{l}\text { Drinking Water } \\
\text { 2(B) (Soft Water) }\end{array}$ & $\begin{array}{l}\text { Forest Water } 1 \\
\text { (Upper Reach) }\end{array}$ & $\begin{array}{l}\text { Forest Water } 2 \\
\text { (Middle Reach) }\end{array}$ & $\begin{array}{l}\text { Forest Water } 3 \\
\text { (Lower Reach) }\end{array}$ \\
\hline $\begin{array}{c}\text { Sampling } \\
\text { Point } \\
\text { Sub-Stances }\end{array}$ & $\begin{array}{l}\text { Kobe City, } \\
\text { Japan }\end{array}$ & $\begin{array}{l}\text { Nancy City, } \\
\text { France }\end{array}$ & $\begin{array}{c}\text { Kameoka City, } \\
\text { Japan }\end{array}$ & $\begin{array}{c}\text { Melbourne } \\
\text { City Australia }\end{array}$ & $\begin{array}{c}\text { Kameoka City, } \\
\text { Japan }\end{array}$ & $\begin{array}{c}\text { Kameoka City, } \\
\text { Japan }\end{array}$ & $\begin{array}{c}\text { Kameoka City, } \\
\text { Japan }\end{array}$ \\
\hline $\mathrm{TH}(\mathrm{mg} / \mathrm{L})$ & $50 \rightarrow 40$ & $1468 \rightarrow 50$ & $30 \rightarrow 10$ & $10 \rightarrow 0$ & $10 \rightarrow 0$ & $20 \rightarrow 0$ & $50 \rightarrow 20$ \\
\hline $\mathrm{pH}$ & $9.0 \rightarrow 9.0$ & $7.4 \rightarrow 7.4$ & $7.5 \rightarrow 7.0$ & $5.0 \rightarrow 5.5$ & $7.5 \rightarrow 7.0$ & $7.5 \rightarrow 7.0$ & $8.0 \rightarrow 7.5$ \\
\hline COD (mg/L) & $8 \rightarrow 0$ & $5 \rightarrow 0$ & $5 \rightarrow 0$ & $5 \rightarrow 0$ & $0 \rightarrow 0$ & $0 \rightarrow 0$ & $4 \rightarrow 0$ \\
\hline $\mathrm{Ca}^{2+}(\mathrm{mg} / \mathrm{L})$ & $35 \rightarrow 25$ & $468 \rightarrow 50$ & $35 \rightarrow 25$ & $5 \rightarrow 0$ & $12.5 \rightarrow 12.5$ & $25 \rightarrow 12.5$ & $50 \rightarrow 25$ \\
\hline $\mathrm{Mg}^{2+}(\mathrm{mg} / \mathrm{L})$ & $31 \rightarrow 20.5$ & $74.5 \rightarrow 41$ & $8.2 \rightarrow 4.1$ & $4.1 \rightarrow 0$ & $0 \rightarrow 0$ & $8.2 \rightarrow 0$ & $8.2 \rightarrow 4.1$ \\
\hline $\mathrm{SiO}_{2}(\mathrm{mg} / \mathrm{L})$ & $0.5 \rightarrow 0.5$ & $8 \rightarrow 5$ & $100 \rightarrow 0$ & $10 \rightarrow 5$ & $15 \rightarrow 15$ & $15 \rightarrow 10$ & $15 \rightarrow 10$ \\
\hline $\mathrm{NH}_{4}^{+} \quad(\mathrm{mg} / \mathrm{L})$ & $0.65 \rightarrow 0.26$ & $0.26 \rightarrow 0.26$ & $0.26 \rightarrow 0.26$ & $0.65 \rightarrow 0.26$ & $0.26 \rightarrow 0.26$ & $0.26 \rightarrow 0.26$ & $0.26 \rightarrow 0.26$ \\
\hline $\mathrm{NO}_{2}^{-} \quad(\mathrm{mg} / \mathrm{L})$ & $0.33 \rightarrow 0.17$ & $0 \rightarrow 0$ & $0.033 \rightarrow 0$ & $0 \rightarrow 0$ & $0 \rightarrow 0$ & $0 \rightarrow 0$ & $0.066 \rightarrow 0.033$ \\
\hline $\mathrm{NO}_{3}^{-} \quad(\mathrm{mg} / \mathrm{L})$ & $8.6 \rightarrow 4.3$ & $2.2 \rightarrow 2.2$ & $8.6 \rightarrow 4.3$ & $8.6 \rightarrow 4.3$ & $0 \rightarrow 0$ & $0.86 \rightarrow 0.86$ & $4.3 \rightarrow 2.2$ \\
\hline $\mathrm{PO}_{4}^{3-} \quad(\mathrm{mg} / \mathrm{L})$ & $0 \rightarrow 0$ & $0 \rightarrow 0$ & $0 \rightarrow 0$ & $0.3 \rightarrow 0.3$ & $0 \rightarrow 0$ & $0.15 \rightarrow 0.06$ & $0.3 \rightarrow 0.15$ \\
\hline BOD (mg/L) & $3 \rightarrow 0$ & $2 \rightarrow 0$ & $3.5 \rightarrow 0$ & $1 \rightarrow 0$ & $1 \rightarrow 0$ & $1.5 \rightarrow 0$ & $2.5 \rightarrow 0$ \\
\hline $\mathrm{Na}^{+}(\mathrm{mg} / \mathrm{L})$ & $7500 \rightarrow 5900$ & $21 \rightarrow 30$ & $6.2 \rightarrow 5.5$ & $8.6 \rightarrow 8.0$ & $5.3 \rightarrow 5.2$ & $5.2 \rightarrow 6.1$ & $9.8 \rightarrow 6.7$ \\
\hline $\mathrm{K}^{+}(\mathrm{mg} / \mathrm{L})$ & $370 \rightarrow 380$ & $4.1 \rightarrow 4.7$ & $1.3 \rightarrow 1.3$ & $1.3 \rightarrow 1.2$ & $0.6 \rightarrow 0.6$ & $0.6 \rightarrow 0.6$ & $2.8 \rightarrow 2.0$ \\
\hline
\end{tabular}

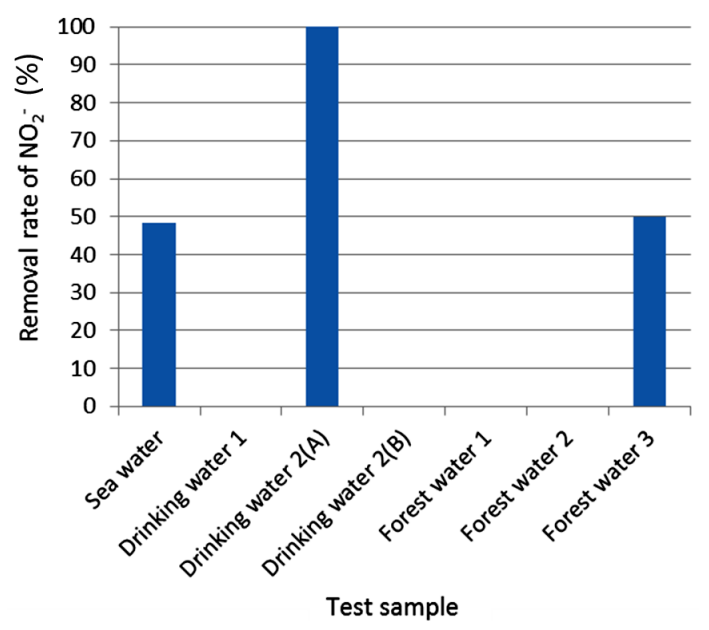

Figure 9. Removal rate of $\mathrm{NO}_{2}^{-}$.

$$
\begin{array}{cc}
\mathrm{Ca}^{2+}+2 \mathrm{NO}_{2}^{-} \rightarrow \mathrm{Ca}\left(\mathrm{NO}_{2}\right)_{2} & \mathrm{Mg}^{2+}+2 \mathrm{NO}_{2}^{-} \rightarrow \mathrm{Mg}\left(\mathrm{NO}_{2}\right)_{2} \\
\mathrm{Ca}^{2+}+2 \mathrm{NO}_{3}^{-} \rightarrow \mathrm{Ca}\left(\mathrm{NO}_{3}\right)_{2} & \mathrm{Mg}^{2+}+2 \mathrm{NO}_{3}^{-} \rightarrow \mathrm{Mg}\left(\mathrm{NO}_{3}\right)_{2} \\
3 \mathrm{Ca}^{2+}+2 \mathrm{PO}_{4}^{3-} \rightarrow \mathrm{Ca}_{3}\left(\mathrm{PO}_{4}\right)_{2} & 3 \mathrm{Mg}^{2+}+2 \mathrm{PO}_{4}^{3-} \rightarrow \mathrm{Mg}_{3}\left(\mathrm{PO}_{4}\right)_{2}
\end{array}
$$

By the way, for distinctive phenomenon, monovalent cations such as $\mathrm{Na}^{+}, \mathrm{K}^{+}$and $\mathrm{NH}_{4}^{+}$show little change after irradiating in all cases. Namely, for example, next reaction never happened.

$$
3 \mathrm{Na}^{+}+\mathrm{PO}_{4}^{3-} \rightarrow \mathrm{Na}_{3} \mathrm{PO}_{4}
$$




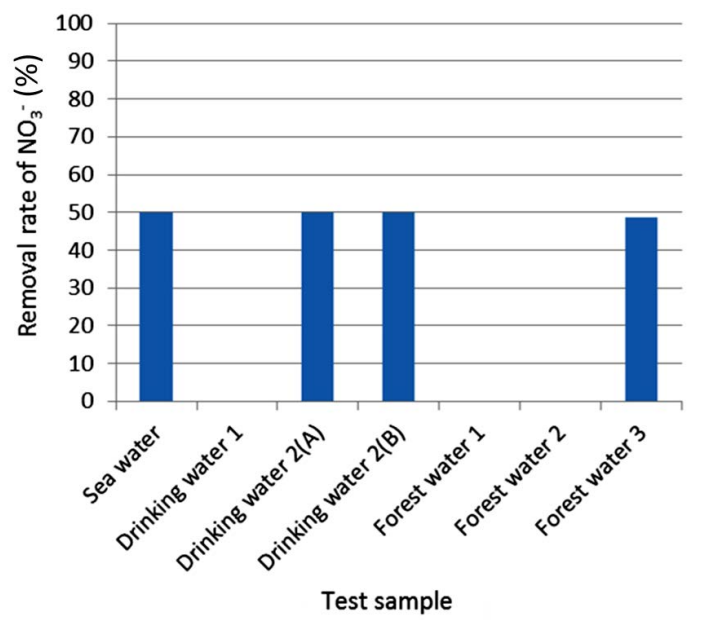

Figure 10. Removal rate of $\mathrm{NO}_{3}^{-}$.

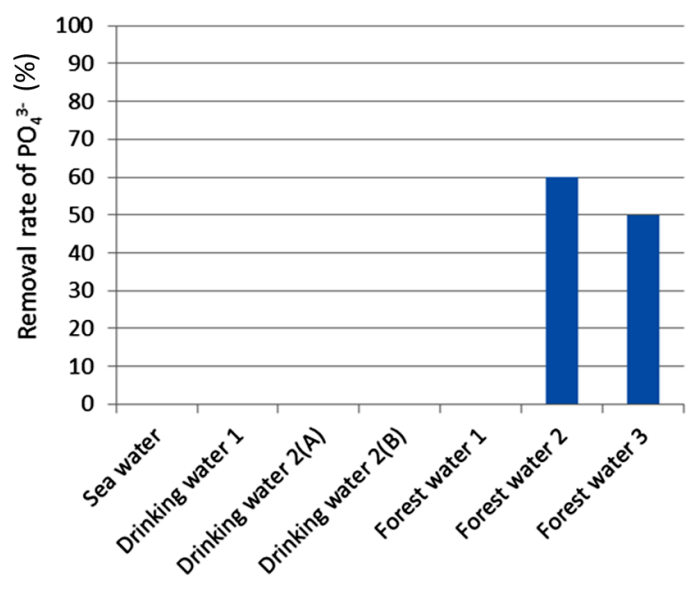

Test sample

Figure 11. Removal rate of $\mathrm{PO}_{4}^{3-}$.

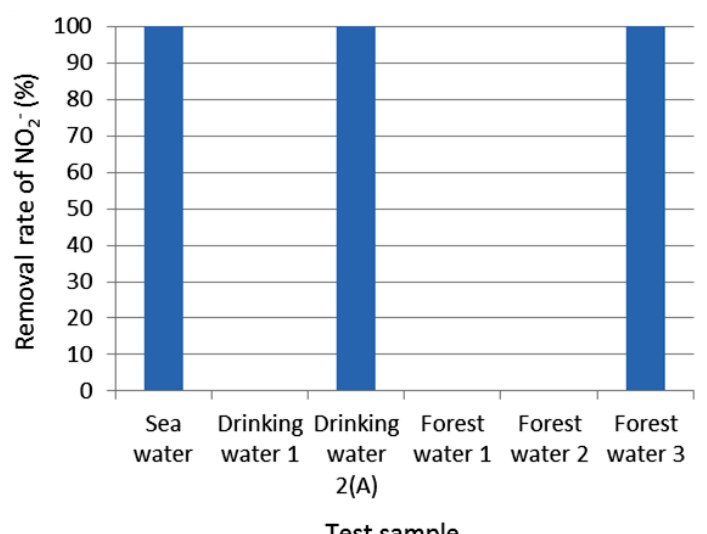

Figure 12. Removal rate of $\mathrm{NO}_{2}^{-}$.

This phenomenon is difficult to understand. We want to clear the mechanism of sound effect on decreasing for $\mathrm{NO}_{2}^{-}\left(\mathrm{NO}_{3}^{-}\right)$and $\mathrm{PO}_{4}^{3-}$ in the near future. 
Table 4. Change of chemical substances in the water by Japanese wind-bell.

\begin{tabular}{|c|c|c|c|c|c|c|c|}
\hline & Sea Water & $\begin{array}{l}\text { Drinking Water } \\
1 \text { (Hard Water) }\end{array}$ & $\begin{array}{l}\text { Drinking Water } \\
\text { 2(A) (Soft Water) }\end{array}$ & $\begin{array}{l}\text { Drinking Water } \\
\text { 2(B) (Soft Water) }\end{array}$ & $\begin{array}{l}\text { Forest Water } 1 \\
\text { (Upper Reach) }\end{array}$ & $\begin{array}{l}\text { Forest Water } 2 \\
\text { (Middle Reach) }\end{array}$ & $\begin{array}{l}\text { Forest Water } 3 \\
\text { (Lower Reach) }\end{array}$ \\
\hline $\begin{array}{c}\text { Sampling } \\
\text { Point } \\
\text { Sub-Stances }\end{array}$ & $\begin{array}{c}\text { Kobe City } \\
\text { Japan }\end{array}$ & $\begin{array}{l}\text { Nancy City } \\
\text { France }\end{array}$ & $\begin{array}{c}\text { Kameoka City } \\
\text { Japan }\end{array}$ & $\begin{array}{c}\text { Melbourne } \\
\text { City Australia }\end{array}$ & $\begin{array}{c}\text { Kameoka City } \\
\text { Japan }\end{array}$ & $\begin{array}{c}\text { Kameoka City } \\
\text { Japan }\end{array}$ & $\begin{array}{c}\text { Kameoka City } \\
\text { Japan }\end{array}$ \\
\hline $\mathrm{TH}$ (mg/L) & $50 \rightarrow 40$ & $1468 \rightarrow 50$ & $30 \rightarrow 10$ & $10 \rightarrow 0$ & $10 \rightarrow 0$ & $20 \rightarrow 0$ & $50 \rightarrow 20$ \\
\hline $\mathrm{pH}$ & $9.0 \rightarrow 9.0$ & $7.4 \rightarrow 7.4$ & $7.5 \rightarrow 7.0$ & $5.0 \rightarrow 5.5$ & $7.5 \rightarrow 7.0$ & $7.5 \rightarrow 7.0$ & $8.0 \rightarrow 7.5$ \\
\hline COD (mg/L) & $8 \rightarrow 0$ & $5 \rightarrow 0$ & $5 \rightarrow 0$ & $5 \rightarrow 0$ & $0 \rightarrow 0$ & $0 \rightarrow 0$ & $4 \rightarrow 0$ \\
\hline $\mathrm{Ca}^{2+}(\mathrm{mg} / \mathrm{L})$ & $35 \rightarrow 25$ & $468 \rightarrow 50$ & $35 \rightarrow 25$ & $5 \rightarrow 0$ & $12.5 \rightarrow 12.5$ & $25 \rightarrow 12.5$ & $50 \rightarrow 25$ \\
\hline $\mathrm{Mg}^{2+}(\mathrm{mg} / \mathrm{L})$ & $31 \rightarrow 20.5$ & $74.5 \rightarrow 41$ & $8.2 \rightarrow 4.1$ & $4.1 \rightarrow 0$ & $0 \rightarrow 0$ & $8.2 \rightarrow 0$ & $8.2 \rightarrow 4.1$ \\
\hline $\mathrm{SiO}_{2}(\mathrm{mg} / \mathrm{L})$ & $0.5 \rightarrow 0.5$ & $8 \rightarrow 5$ & $100 \rightarrow 0$ & $10 \rightarrow 5$ & $15 \rightarrow 15$ & $15 \rightarrow 10$ & $15 \rightarrow 10$ \\
\hline $\mathrm{NH}_{4}^{+} \quad(\mathrm{mg} / \mathrm{L})$ & $0.65 \rightarrow 0.26$ & $0.26 \rightarrow 0.26$ & $0.26 \rightarrow 0.26$ & $0.65 \rightarrow 0.26$ & $0.26 \rightarrow 0.26$ & $0.26 \rightarrow 0.26$ & $0.26 \rightarrow 0.26$ \\
\hline $\mathrm{NO}_{2}^{-} \quad(\mathrm{mg} / \mathrm{L})$ & $0.33 \rightarrow 0.17$ & $0 \rightarrow 0$ & $0.033 \rightarrow 0$ & $0 \rightarrow 0$ & $0 \rightarrow 0$ & $0 \rightarrow 0$ & $0.066 \rightarrow 0.033$ \\
\hline $\mathrm{NO}_{3}^{-} \quad(\mathrm{mg} / \mathrm{L})$ & $8.6 \rightarrow 4.3$ & $2.2 \rightarrow 2.2$ & $8.6 \rightarrow 4.3$ & $8.6 \rightarrow 4.3$ & $0 \rightarrow 0$ & $0.86 \rightarrow 0.86$ & $4.3 \rightarrow 2.2$ \\
\hline $\mathrm{PO}_{4}^{3-} \quad(\mathrm{mg} / \mathrm{L})$ & $0 \rightarrow 0$ & $0 \rightarrow 0$ & $0 \rightarrow 0$ & $0.3 \rightarrow 0.3$ & $0 \rightarrow 0$ & $0.15 \rightarrow 0.06$ & $0.3 \rightarrow 0.15$ \\
\hline BOD (mg/L) & $3 \rightarrow 0$ & $2 \rightarrow 0$ & $3.5 \rightarrow 0$ & $1 \rightarrow 0$ & $1 \rightarrow 0$ & $1.5 \rightarrow 0$ & $2.5 \rightarrow 0$ \\
\hline $\mathrm{Na}^{+}(\mathrm{mg} / \mathrm{L})$ & $7500 \rightarrow 5900$ & $21 \rightarrow 30$ & $6.2 \rightarrow 5.5$ & $8.6 \rightarrow 8.0$ & $5.3 \rightarrow 5.2$ & $5.2 \rightarrow 6.1$ & $9.8 \rightarrow 6.7$ \\
\hline $\mathrm{K}^{+}(\mathrm{mg} / \mathrm{L})$ & $370 \rightarrow 380$ & $4.1 \rightarrow 4.7$ & $1.3 \rightarrow 1.3$ & $1.3 \rightarrow 1.2$ & $0.6 \rightarrow 0.6$ & $0.6 \rightarrow 0.6$ & $2.8 \rightarrow 2.0$ \\
\hline
\end{tabular}

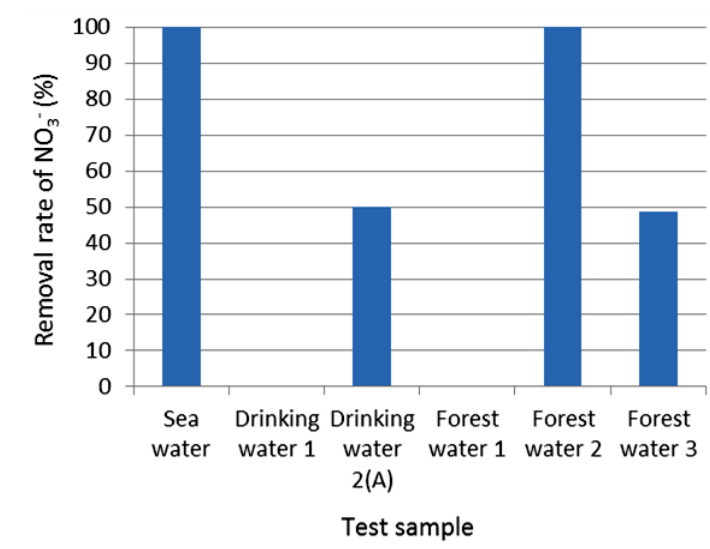

Figure 13. Removal rate of $\mathrm{NO}_{3}^{-}$.

\subsection{Comparison of an Effect of Each Sound Wave for Water Quality Improvement}

Figure 15 and Figure 16 show a removal rate of $\mathrm{NO}_{2}^{-}$and $\mathrm{PO}_{4}^{3-}$ by each sound wave respectively. From these figures, sound waves with a wide range of frequency have effect on improvement of water quality. Sound wave is transmitted in the transverse manner with repeating expansion and compression. It is believed that expansion and compression agitates the fluid and facilitates the reaction mentioned above which means boundary film thickness in Equation (1) is made thin. In this study, the threshold values of the work on the water improvement generated by sound wave are not obtained. It is the theme in the near future.

$$
-\mathrm{d}(C) / \mathrm{d} t=V / \delta\left([C]-\left[C_{0}\right]\right)
$$

where 


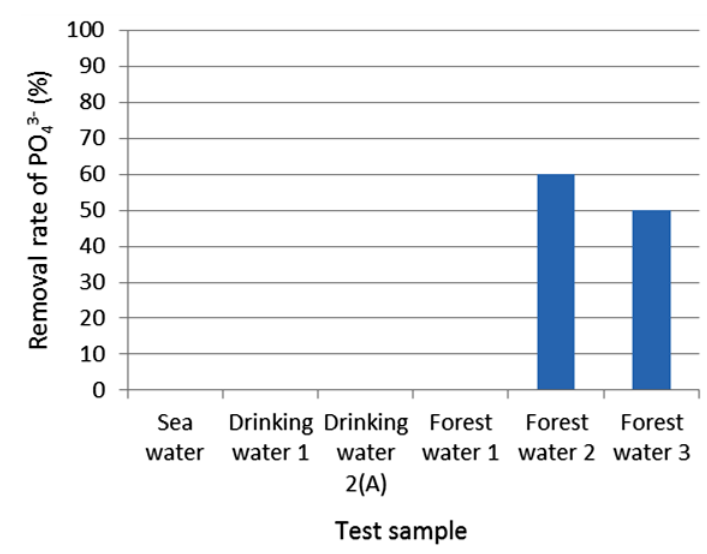

Figure 14. Removal rate of $\mathrm{PO}_{4}^{3-}$.

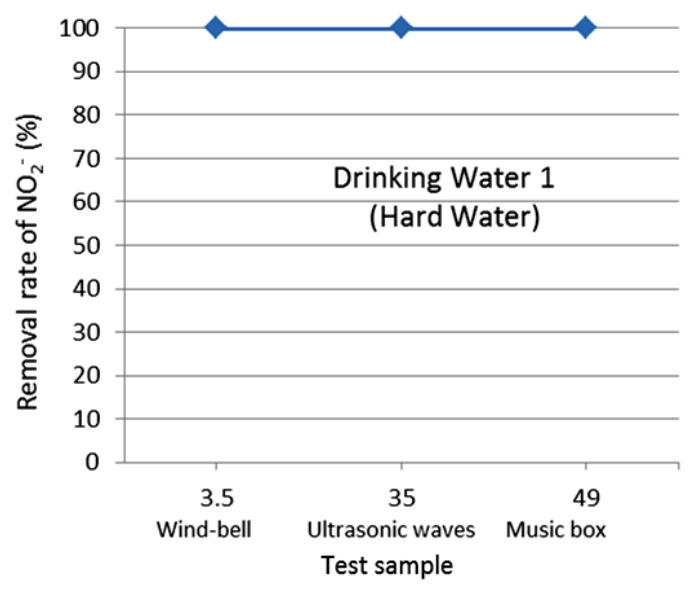

Figure 15. Removal rate of $\mathrm{NO}_{2}^{-}$.

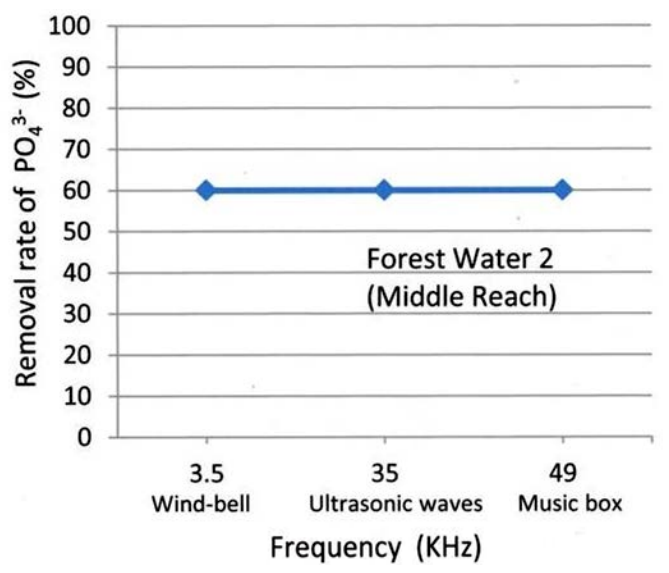

Figure 16. Removal rate of $\mathrm{PO}_{4}^{3-}$.

C: concentration (mg/L);

$C_{0}$ : Initial concentration $(\mathrm{mg} / \mathrm{L})$;

$t$ : time (min.);

$V$ : volume (ml);

$\delta$ : boundary film thickness (mm). 
Furthermore, from the results mentioned above, a possibility of the improvement of water quality by murmuring sound of a natural stream was suggested. If such a phenomenon may occurs, it is confirmed that natural purification mechanism could work. This is a subject for the near future.

\section{Conclusions}

In this study, various sound waves such as 1) ultrasonic waves, 2) music box, and 3) wind-bell were irradiated to water and wastewater for removing contaminants such as nitrate, phosphorus and BOD/COD. The conclusion of the paper is as follows.

1) Various sound waves affect on the improvement of the water quality.

2) As for the removal rate of $\mathrm{NO}_{2}^{-}$and $\mathrm{NO}_{3}^{-}$concerning to the three samples such as the seawater, drinking water and the forest water, high values ( $>50 \%)$ were obtained, respectively.

3) $\mathrm{PO}_{4}^{3-}$ was also confirmed to decrease more than $50 \%$ in the forest water.

4) $\mathrm{BOD}$ and $\mathrm{COD}$ was drastically decreased especially in drinking water and forest water.

5) The removal rate of $\mathrm{Ca}^{2+}$ and $\mathrm{Mg}^{2+}$ was also large (>50\%) in the drinking water. Along with these, $\mathrm{TH}$ also drastically decreased. On the other hand, $\mathrm{Na}^{+}, \mathrm{K}^{+}$and $\mathrm{NH}_{4}^{+}$showed very few change.

6) Therefore, decreases of $\mathrm{NO}_{2}^{-}, \mathrm{NO}_{3}^{-}$and $\mathrm{PO}_{4}^{3-}$ are due to the reaction with bivalent cations of $\mathrm{Ca}^{2+}$ and $\mathrm{Mg}^{2+}$ rather than monovalent cations such as $\mathrm{Na}^{+}, \mathrm{K}^{+}$and $\mathrm{NH}_{4}^{+}$.

7) It is supposed that if hard water with components of $\mathrm{Ca}^{2+}$ and $\mathrm{Mg}^{2+}$ is properly added into a soft drinking water, and the sound waves are irradiated to them, $\mathrm{NO}_{2}^{-}, \mathrm{NO}_{3}^{-}$and $\mathrm{PO}_{4}^{3-}$ could be reduced. It could be useful from the industrial viewpoint.

\section{Acknowledgements}

The authors are very grateful to Dr. Pathak D.R. for his insightful comments and excellent assistance on the manuscript.

\section{References}

[1] Pathak, D.R., Hiratsuka, A. and Yamashiki, Y. (2011) Influence of Anthropogenic Activities and Seasonal Variation on Groundwater Quality of Kathmandu using Multivariate Statistical Analysis. In: Peters, N.J., et al., Eds., Assessment of Water Quality under Changing Climate Conditions, Proceedings of Symposium H04 Held during IUGG2011, IAHS Red Book, Melbourne, Volume 348, 67-72.

[2] Kostraba, J.N., Gay, E.C., Rewers, M. and Hamman, R.F. (1992) Nitrate Levels in Community Drinking Water and Risk of IDDM-An Ecological Analysis. Diabetes Care, 15, 1505-1508.

[3] Gaya, U.I. and Abdullah, A.H. (2008) Heterogeneous Photocatalytic Degradation of Organic Contaminants over Titanium Dioxide: A Review of Fundamentals, Progress and Problems. Journal of Photochemistry and Photobiology C: Photochemistry Reviews, 9, 1-12. http://dx.doi.org/10.1016/j.jphotochemrev.2007.12.003

[4] Chong, M.N., Jin, B. and Chow, C.W.K. (2010) Recent Developments in Photocatalytic Water Treatment Technology: A Review. Water Research, 44, 2997-3027. http://dx.doi.org/10.1016/j.watres.2010.02.039

[5] Hiratsuka, A. and Pathak, D.R. (2013) Application of Ultrasonic Waves for the Improvement of Water Treatment. Journal of Water Resource and Protection, 5, 604-610. http://dx.doi.org/10.4236/jwarp.2013.56061 
Scientific Research Publishing (SCIRP) is one of the largest Open Access journal publishers. It is currently publishing more than 200 open access, online, peer-reviewed journals covering a wide range of academic disciplines. SCIRP serves the worldwide academic communities and contributes to the progress and application of science with its publication.

Other selected journals from SCIRP are listed as below. Submit your manuscript to us via either submit@scirp.org or Online Submission Portal.
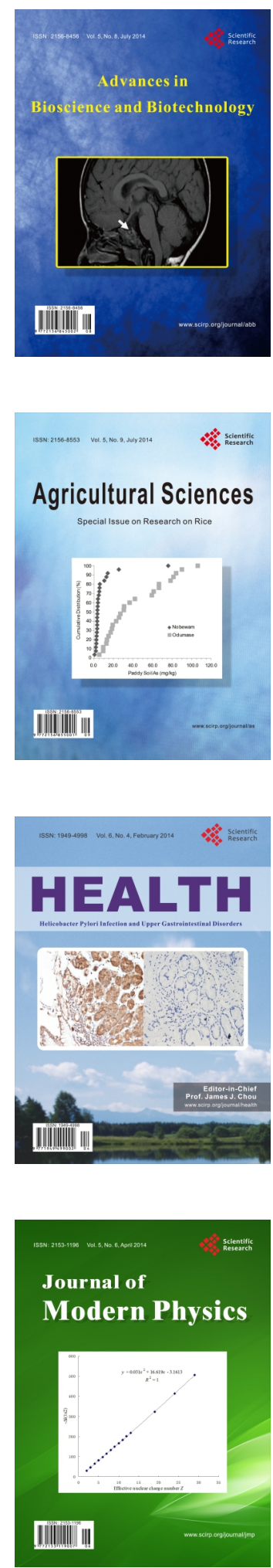
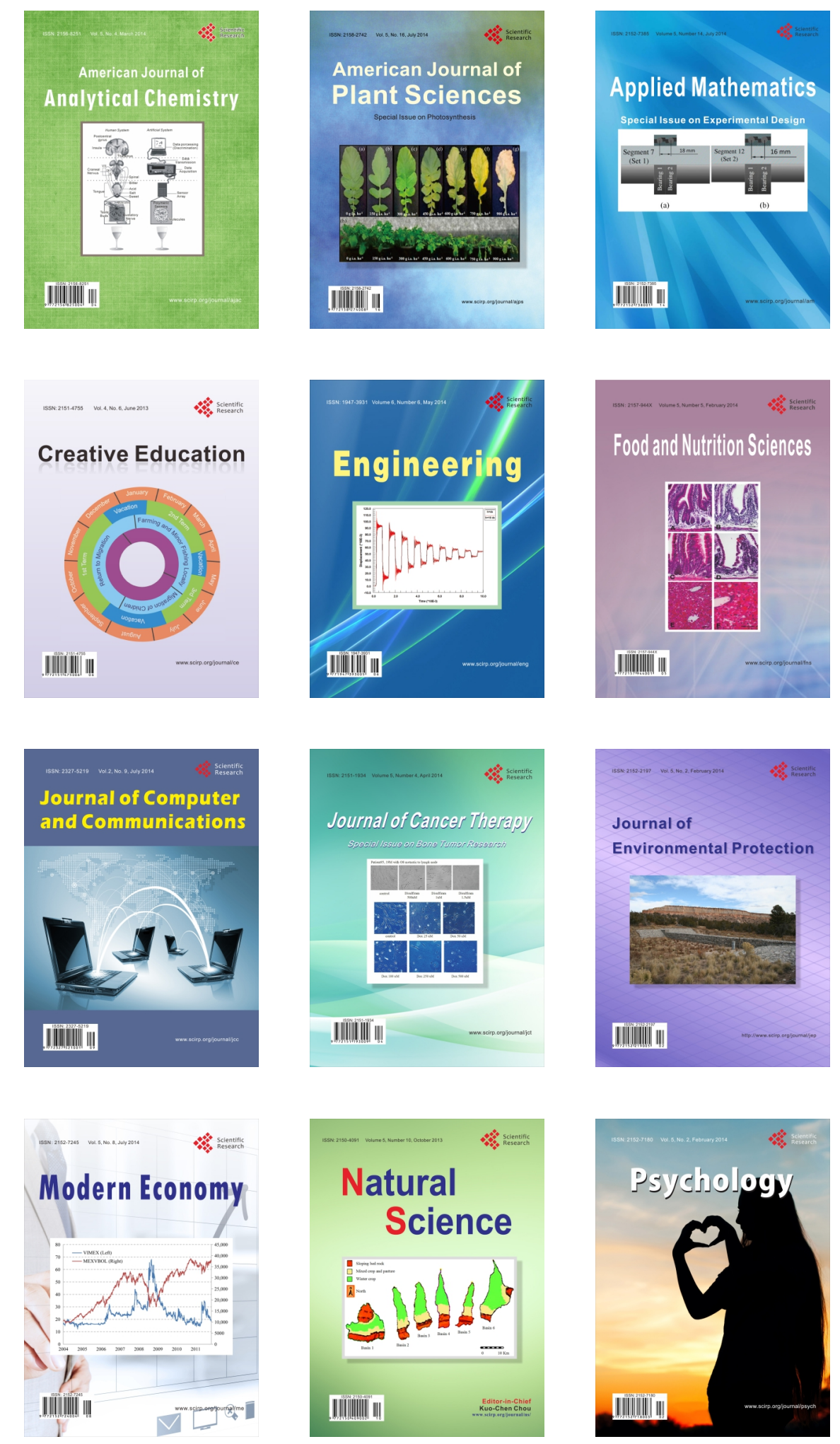